\title{
Comparison of the Rigid Rod System with Modular Plate with the Finite Element Analysis in Short-Segment Posterior Stabilization in the Lower Lumbar Region
} \author{
Onur YAMAN ${ }^{4}$, Insan SOLAROGLU ${ }^{4}$, Fahir OZER ${ }^{4}$ \\ 'Sakarya Training and Research Hospital, Department of Neurosurgery, Sakarya, Turkey \\ ${ }^{2}$ Alanya State Hospital, Department of Neurosurgery, Antalya, Turkey \\ ${ }^{3}$ Sincan State Hospital, Department of Neurosurgery, Ankara, Turkey \\ ${ }^{4} \mathrm{Koc}$ University, School of Medicine, Department of Neurosurgery, Istanbul, Turkey \\ ${ }^{5} \mathrm{Koc}$ University, School of Medicine, Department of Mechanical Engineering, Istanbul, Turkey
}

Can YALDIZ ${ }^{1}$, Birol OZKAL ${ }^{2}$, Yahya GUVENC ${ }^{3}$, Salim SENTURK ${ }^{4}$, Deniz ERBULUT' ${ }^{5}$, Iman ZAFARPARANDAH ${ }^{5}$,

\section{ABSTRACT}

AIM: Many studies are available in the literature on posterior spinal instrumentation, though the use of a rod and a plate is still controversial in the literature. In this study, a finite element analysis of the strength and superiority of modular rigid plate and rod systems, which are used in the lower lumbar region, in comparison with each other was used.

MATERIAL and METHODS: A Ti6AI4V (Grade 5) titanium biocompatible alloy anterior plate was used for the lumbar spine fixation device, and a finite element analysis was conducted on the human lumbar spine model. In this study, an intact spine, a rigid system fixed with a rod, and modular plate systems were evaluated at flexion, extension, lateral bending, and axial rotation.

RESULTS: They did not show statistically significant superiority over one another in terms of limitations in movement during the range of motion exercises and rigidity.

CONCLUSION: The posterior rigid stabilization system and novel stabilization system do not have a significant superiority over one another. Equivalent results in the limitation of movement and rigidity allow for the use of these systems for short-segment posterior spinal instrumentation with the same indications.

KEYWORDS: Biomedical device, Finite element model, Joint biomechanics, Musculo-skeletal mechanics, Orthopedic material

\section{INTRODUCTION}

$\mathrm{T}$ Today, posterior spinal stabilization and fusion interventions occupy an important place in neurosurgical practice. Spinal fusion and instrumentation are often applied to provide stability for an unstable spine, to avoid injury in the neurological structure, to reduce incorrect alignment and deformities, to increase the probability of fusion, and to reduce pain in the long term (8). Although this procedure has a very high success rate, the mechanism of the procedure can lead to some limitations in the long term. The biomechanics of the spine are also changing, because it creates non-physiological conditions by blocking movement (16).

The idea of fusion in spinal surgery was raised for the first time at the beginning of the last century. When looking at the literature, it has been shown that the importance of spinal fusion was not exactly known before this period, and surgeons were content with fixation. Thereafter, the first fixation in the thoracolumbar spine by using wire was performed by Hadra 
in 1889, and this was followed by a wire fixation due to a C6-7 dislocation (9). In 1911, the first spinal fusion was achieved by two different surgeons named Albee (1) and Hibbs (10). When reviewing the development of lumbar fusion, it can be seen that Capaner performed the first ALIF surgery for spondylolisthesis in 1932. In 1940, Cloward (4) performed the first PLIF surgery. In 1939, Venable and Stuck (20) proposed the use of Vitallium for internal fixation. In 1942, William Rogers fixed the spinous process with wire in case the reduction was applied with traction (18). In 1944, King defined the facet screwing technique for the first time (12). In 1959, Boucher moved the screw deeper toward the pedicle to develop this technique (2). In 1961, Humphries used a plate in the lumbosacral region to strengthen the anterior fusion with an anterior plate (11). In later years, Roy-Camille (17) and Louise (14) used pedicle screws and plates. In the 1980s, Magerl (15), Steffe et al. (19), Krag et al. (13), Edwards (6), Zielke et al. (22), and Cotrel and Debousset (5) used many different systems with rods that combine the pedicle and the hook. Nowadays, the most accepted method is stabilization with transpedicular screw systems and, if necessary, anterior surgery.

Many studies are available in the literature on posterior spinal instrumentation, though the use of a rod and a plate is still controversial in the literature. In this study, a finite element (FE) analysis of the strength and superiority of modular rigid plate and rod systems, which are used in the lower lumbar region, in comparison with each other was used.

\section{MATERIAL and METHODS}

A Ti6Al4V (Grade 5) titanium biocompatible alloy anterior plate for lumbar spine fixation device was used, and an FE analysis of the human lumbar spine model was applied.

The objectives of this analysis were to determine the range of motion (ROM) of the index and adjacent levels after the implantation of a novel stabilization system (NSS) and to compare with intactness and intactness with standard posterior rigid stabilization systems (PRSSs). The FE analysis conditions were as follows:
1. Intact lumbar spine

2. Intact lumbar spine + novel stabilization system

3. Intact lumbar spine + standard posterior rigid stabilization system

The two end parts of a module were a tongue and a groove, so that the modules could be attached to each other (Figure $1 \mathrm{~A}, \mathrm{~B})$.

\section{Finite Element Model}

Geometry: The geometry of the lumbar spine was obtained from computed tomography (CT) data of a healthy 35-yearold man. The CT data were processed with medical image processing software (Mimics $₫$ Version 14.1; Materialise, Inc., Leuven, Belgium), and a three-dimensional surface definition of the vertebrae L1 to the sacrum was created. The lumbar lordosis was measured between L1-L5 and it was $25^{\circ}$. The detailed mesh procedure can be found in the authors' previous study. The material properties, load and boundary conditions and simulations were performed using the Abaqus software program (Abaqus $\AA$, Version 6.10-2; Abaqus, Inc., Providence, $\mathrm{RI}, \mathrm{USA}$ ).

Vertebra: The vertebrae mesh was divided into three parts: the cortical layer, cancellous core, and posterior elements. An elastic isotropic constitutive model was used to simulate the material behaviors of the elements on the vertebrae.

Intervertebral disc: The intervertebral disc, comprising an annulus fibrosis and nucleus pulposus, was modeled as a composite structure. A circular mesh pattern was generated on the discs in mesh steps to mimic the circular layers of the annulus (Figure 2). The Neo-Hookean hyperelastic model was used to simulate the material behavior of the annulus ground substance. The inner part was defined as the nucleus pulposus and it comprised approximately $40 \%$ of all the elements in the disc area. The fluid behavior of the nucleus was simulated by assigning a very low stiffness $(1 \mathrm{MPa})$ and near incompressibility (Poisson ratio, $v=0.4999$ ). The rebar option of the Abaqus software was used to simulate the fiber behavior and reinforce the annulus ground substance. The rebars were oriented at $\pm 25^{\circ}$ to the horizontal plane, and the
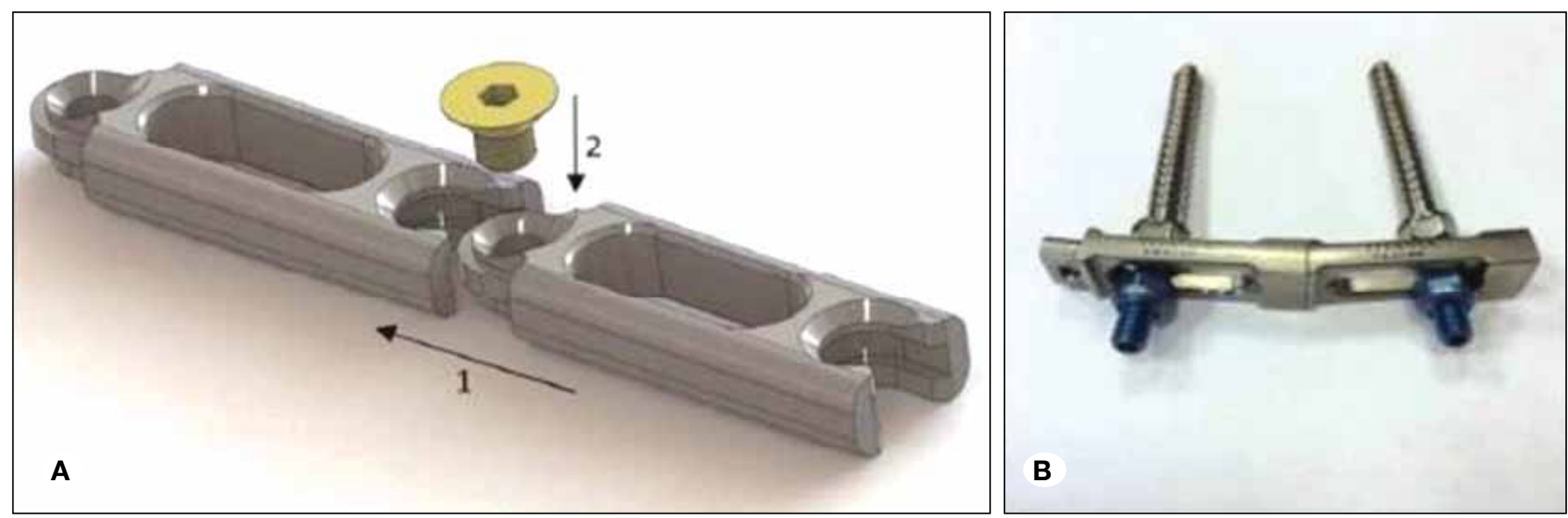

Figure 1: A novel anterior plate for a lumbar spine fixation device. A) assembly direction, B) two parts fixation with a screw. 
"no compression" option was used to mimic the behaviors of the fibers in tension.

Ligament: Five groups of ligaments were simulated in each segment: the anterior longitudinal ligament (ALL), posterior longitudinal ligament (PLL), ligamentum flavum (LF), capsular ligament (CL), and interspinous ligament (ISL). The behavior of the ligaments was modeled using truss elements with the "no-compression" option of the Abaqus software. The number of elements for each group was chosen in such a manner that the entire cross-sectional area of each group matches the experimental values reported in the literature (22). The hypoelastic constitutive model was used to simulate the changes in the stiffness of the ligaments (low stiffness in low strains and high stiffness in high strains).

Facet joint: Facet joints were simulated using GAPUNI elements. The cartilaginous layer on the bone was modeled by a "softened contact" parameter available in the Abaqus software. This parameter exponentially adjusts the force transfer across the joint depending on the gap size.

\section{Boundary and Loading Conditions}

In all directions, the nodes lying on the upper endplate of L1 were coupled to a flying node (FN) higher than the surface of the L1 endplate; then, a pure moment was applied to the FN. The follower load was applied on each side of all segments such that the unwanted segmental rotation was less than $0.2^{\circ}$ (19). The follower load was simulated using the connector elements between each set of adjacent vertebrae. The nodes lying at the bottom endplate of L5 were constrained in all directions.
A 10-Nm bending moment was applied to the superior surface of the L1 vertebra in the intact spine, and the segmental and overall ROM were obtained in flexion (Flex), extension (Ext), lateral bending (LB), and axial rotation (AR). The follower load concept was used to apply $400 \mathrm{~N}$ as the body weight in each segment.

\section{RESULTS}

The L1-L5 segmental ROM is presented in Figures 2 and 3 of intactness and intactness with instrumentation for FE modeling. The FE analysis predicted the ROM for intactness and intactness with an instrumented FE lumbar spine. In PRSS, in Flex, L3-L4: 3.6017, L4-L5: 0.5756, and L5-S1: 8.5471; in Ext, L3-L4: 3.7398, L4-L5: 0.1551, and L5-S1: 2.8675; in LB, L3-L4: 7.3486, L4-L5: 0.0159, and L5-S1: 7.2591; and in AR, L3-L4: 3.7418, L4-L5: 0161, and L5-S1: 4.6354. In NSS, in Flex, L3-L4: 3.6075, L4-L5: 0.6194, and L5-S1: 8.5204; in Ext, L3-L4: 3.8015, L4-L5: 0.1792, and L5-S1: 2.8733; in LB, L3-L4: 7.4565, L4-L5: 0.0056, and L5-S1: 7.4067; in AR, L3L4: 3887, L4-L5: 0.3046, and L5-S1: 4.2563 (Table I).

The ROM of intactness with an instrumented FE model in \% of intactness was as follows. In PRSS, in Flex, L3-L4: 3.1 L4L5: 88.5, and L5-S1: 1.0; in Ext, L3-L4: 2.8, L4-L5: 95.2, and L5-S1: 3.1; in LB, L3-L4: 0.0, L4-L5: 99.8, and L5-S1: 0.5; and in AR, L3-L4: 1.1, L4-L5: 95.9, and L5-S1: 0.1. In NSS, in Flex, L3-L4: 3.2, L4-L5: 87.7, and L5-S1: 0.7; in Ext, L3-L4: 4.5, L4-L5: 94.4, and L5-S1: 2.9; in LB, L3-L4: 1.4, L4-L5: 99.9, and L5-S1: 1.5; and in AR, L3-L4: 2.8, L4-L5: 92.2, and L5-S1: 8.3 (Table II).

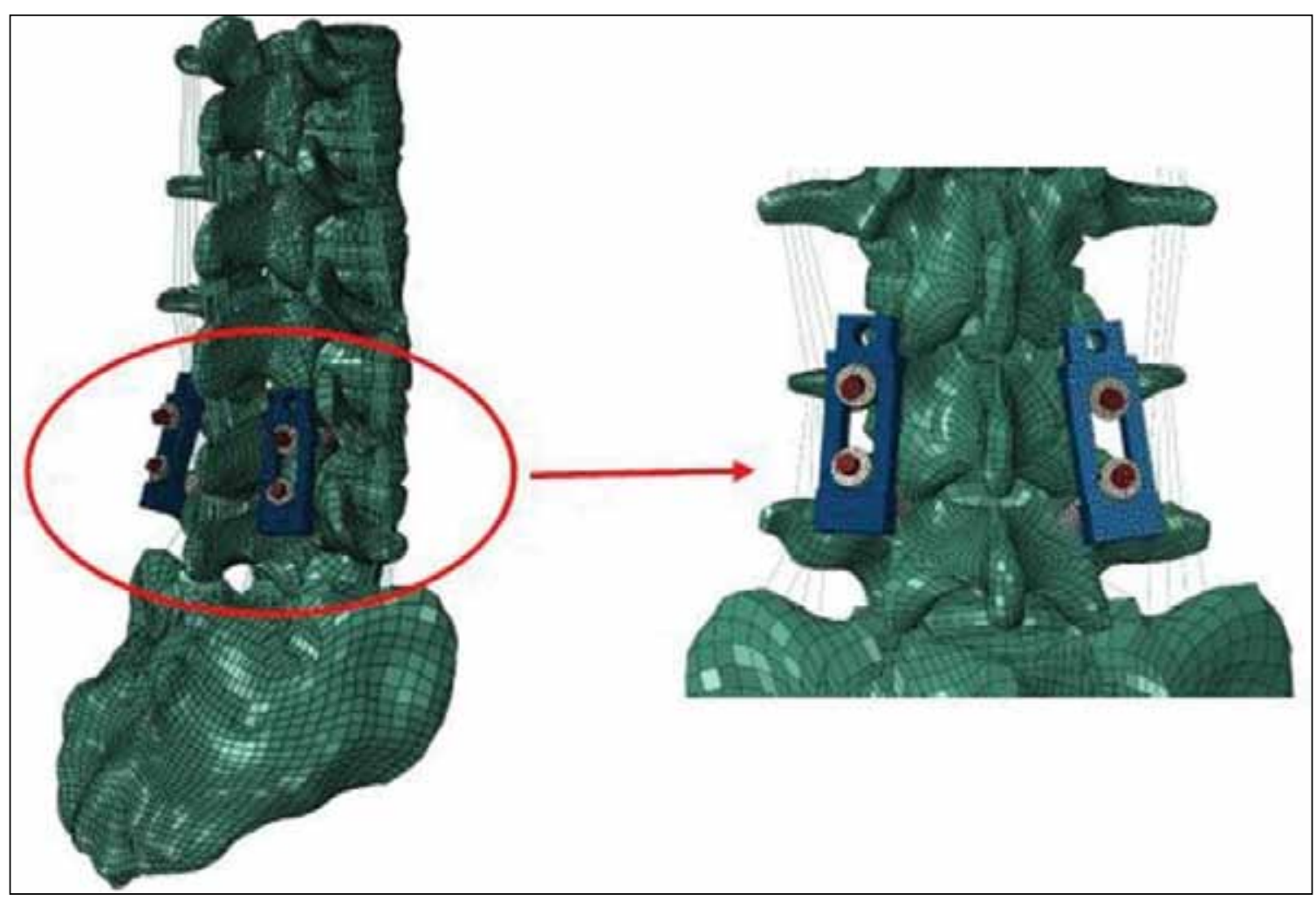

Figure 2: FE model of the lumbar spine with a pediclebased posterior plate stabilization system. 
The ROM of intactness and intactness with an instrumented FE model and the ROM of intactness with an instrumented FE model in \% of intactness in Flex, Ext, LB, and AR are shown in Figures 4A-D, 5A-D.

\section{DISCUSSION}

The main objective of the posterior spinal fusion surgery is to create a safe spinal alignment and an adequate fusion. The choice of instrumentation and required spinal fusion levels are determined as a result of planning before the surgery. For effective fixation, the pedicle, which is the strongest part of the vertebra, is used, and the screw is advanced until the vertebral body. It has been shown in biomechanical studies that the fixation force provided by pedicle screws was superior to other methods. The force is distributed over several segments with the segmental use of a pedicle screw, and the load is reduced for each segment. Thus, early mobilization of the patient is achieved. The breaking of the rods, loosening or breaking of the transpedicular screw, and dislocation of the transverse connectors can be seen as instrumentation problems (7), and it is usually a sign of pseudoarthrosis. If fusion developed, treatment is not required (20).

There are two types of displacements of the lumbar spine in all three action plans, including separate translation and

Table I: ROM of Intactness and Intactness with an Instrumented FE Lumbar Spine

\begin{tabular}{ccccccc}
\hline & \multicolumn{3}{c}{ Flexion } & \multicolumn{3}{c}{ Extension } \\
\hline L1-L2 & Intact & Rod-Fusion & Plate-Fusion & Intact & Rod-Fusion & Plate-Fusion \\
\hline L2-L3 & 3.2349 & 3.3013 & 3.3223 & 4.0983 & 4.1376 & 4.1315 \\
\hline L3-L4 & 3.8944 & 3.9982 & 4.0021 & 2.8547 & 2.8988 & 2.9671 \\
\hline L4-L5 & 3.4941 & 3.6017 & 3.6075 & 3.6365 & 3.7398 & 3.8015 \\
\hline L5-S1 & 5.0175 & 0.5756 & 0.6194 & 3.2191 & 0.1551 & 0.1792 \\
\hline & 8.4644 & 8.5471 & 8.5204 & 2.9593 & 2.8675 & 2.8733 \\
\hline L1-L2 & 7.1531 & 7.0411 & 7.037 & 3.4064 & 3.4535 & 3.967 \\
\hline L2-L3 & 6.4113 & 6.4119 & 6.4952 & 3.354 & 3.372 & 3.7462 \\
\hline L3-L4 & 7.3501 & 7.3486 & 7.4565 & 3.7829 & 3.7418 & 3.887 \\
\hline L4-L5 & 7.0512 & 0.0159 & 0.0056 & 3.9243 & 0.161 & 0.3046 \\
\hline L5-S1 & 7.2952 & 7.2591 & 7.4067 & 4.6391 & 4.6354 & 4.2563 \\
\hline
\end{tabular}

Table II: ROM of Intactness with an Instrumented FE Model in \% of Intactness

Flexion

Extension

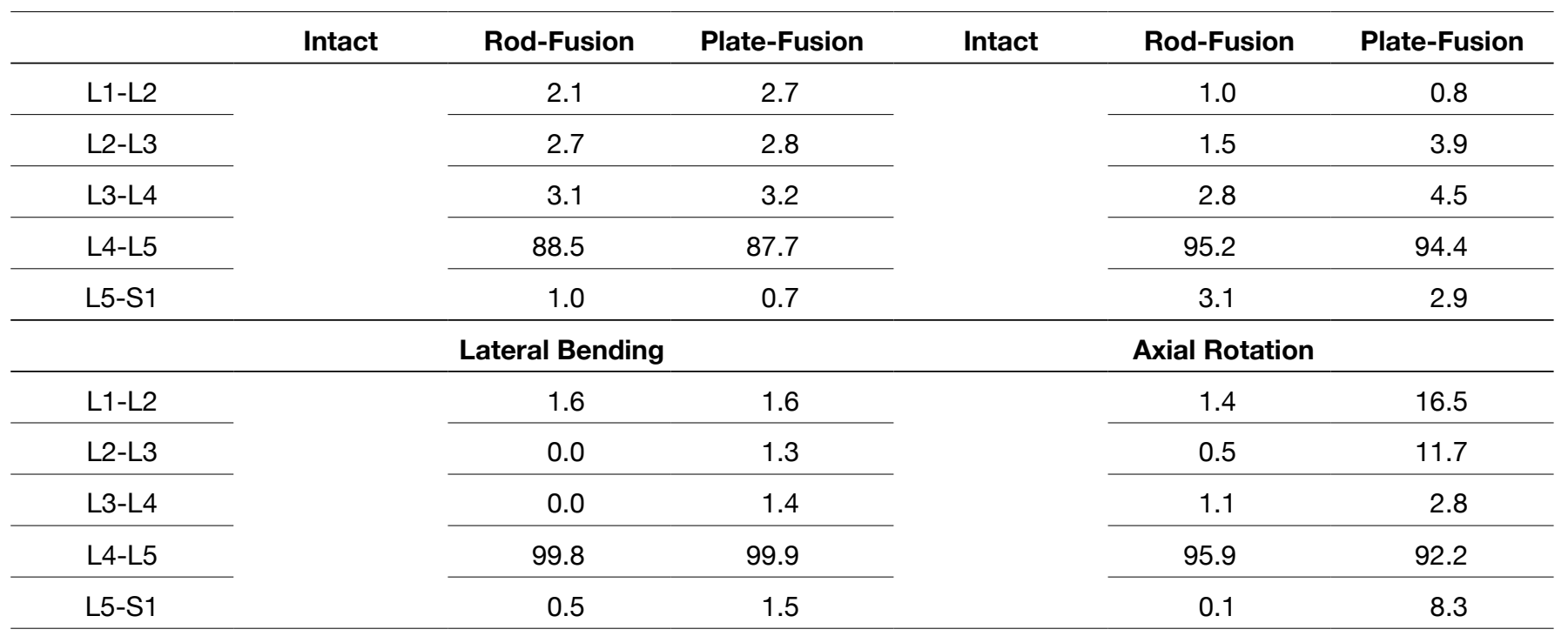




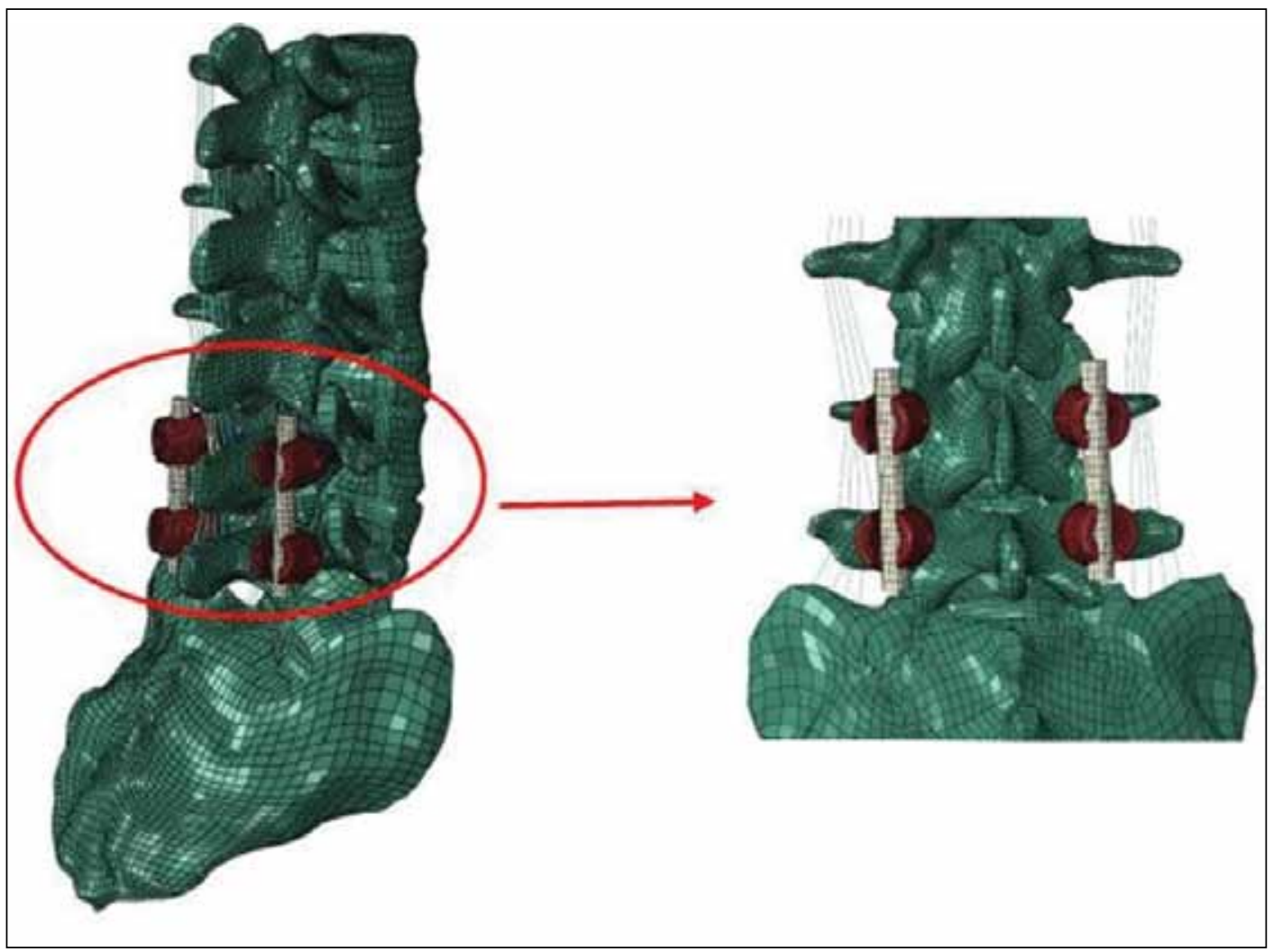

Figure 3: FE model of the lumbar spine with a normal pedicle-based posterior stabilization system.
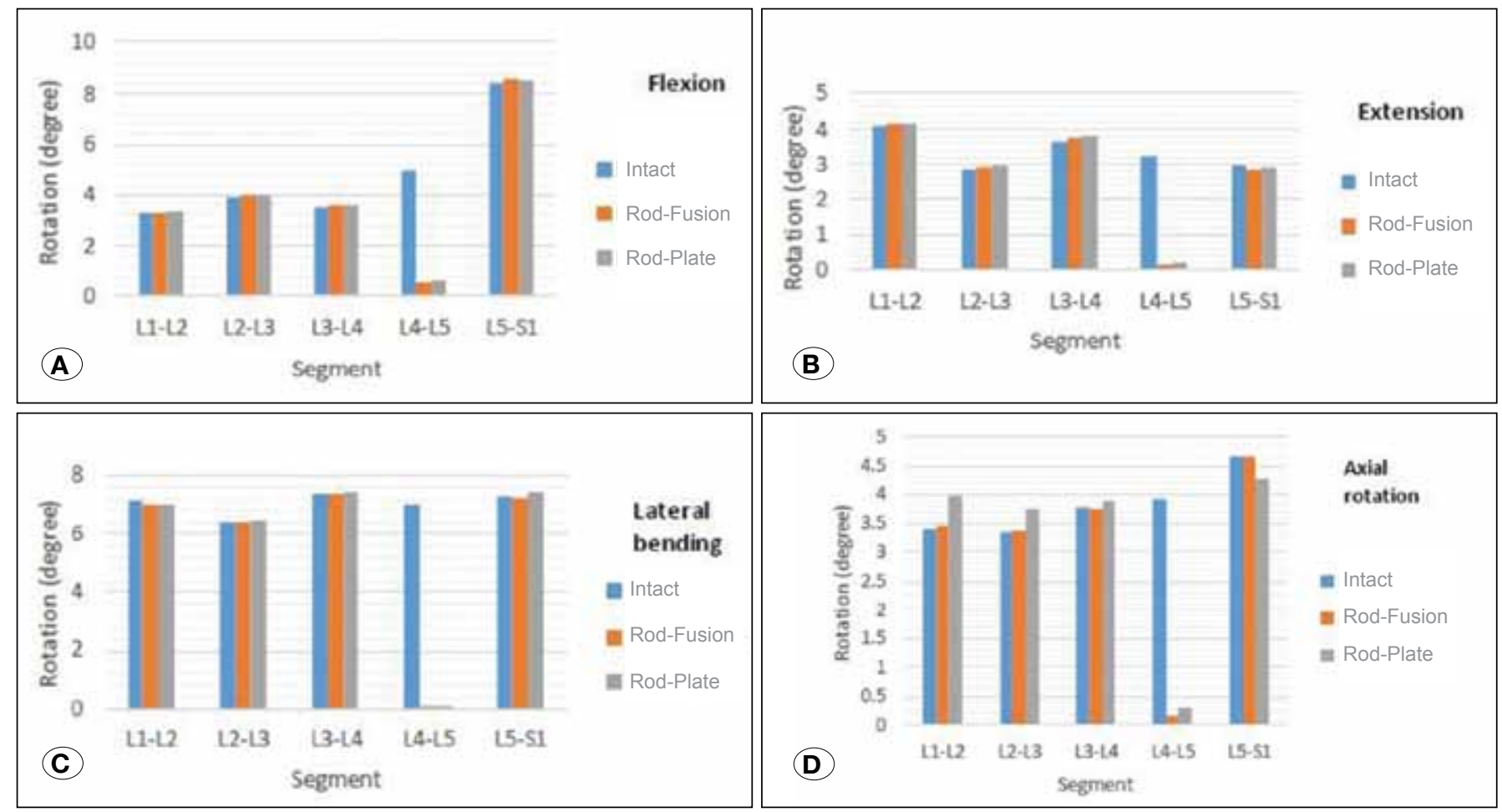

Figure 4: ROM of intactness and intactness with an instrumented FE model in A) Flexion, B) Extension, C) Lateral bending, and D) Axial rotation. 


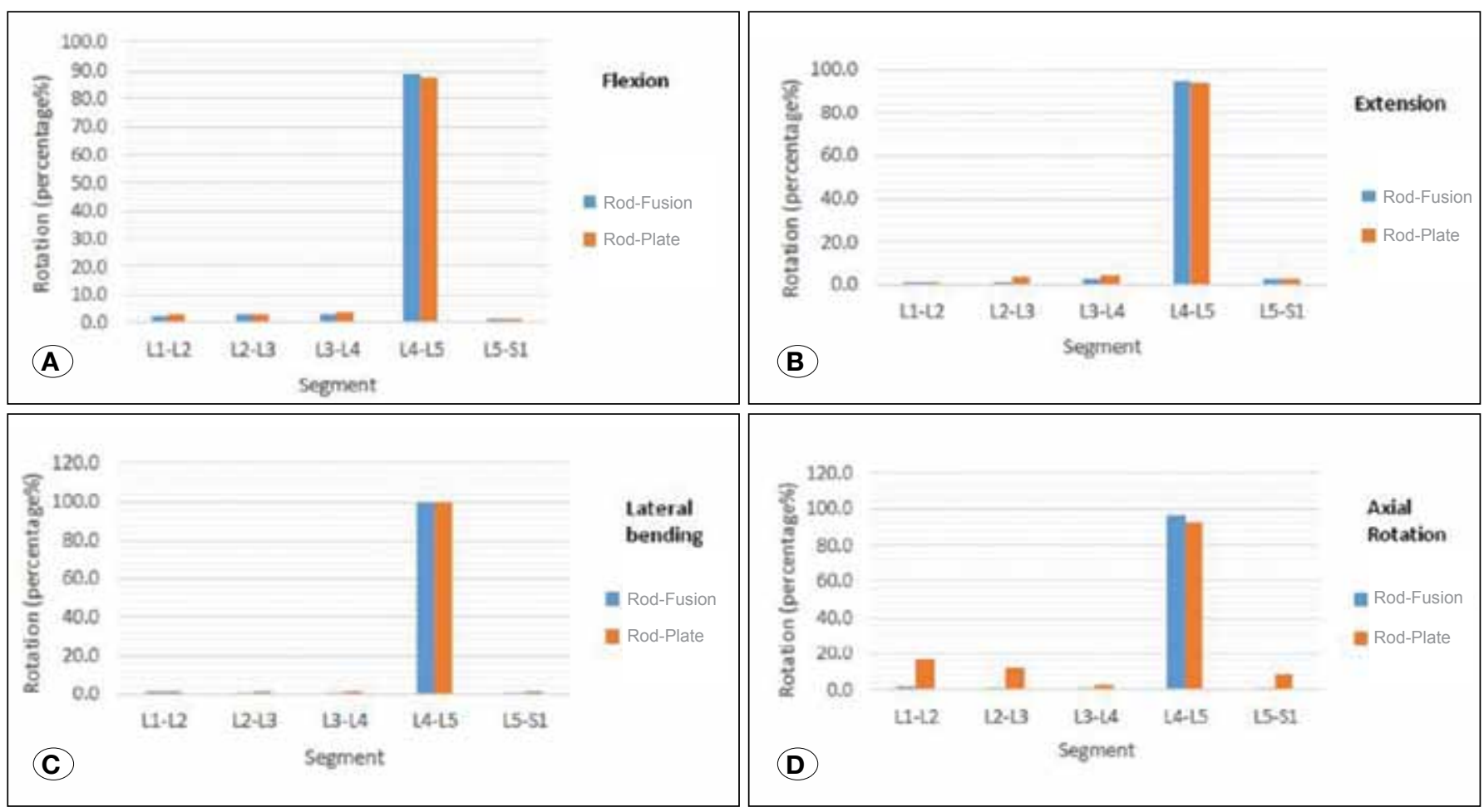

Figure 5: ROM of intactness with an instrumented FE model in \% of intactness in A) Flexion, B) Extension, C) Lateral bending, and D) Axial rotation.

angulation. Thus, the spinal column and any part of it can achieve six different movements. The growth of the vertebra of the spine by moving towards the distal region, showing the physiological curvature for four times, and the anatomical features of the bone and soft tissue structures in accordance with the curvature are important in terms of spinal movements and the transport of the load over the spine. In the lumbar region, the movements of $60^{\circ}$ of Flex and $35^{\circ}$ of Ext are seen. Lateral Flex and the rotational movements are seen in the lumbar region as $20^{\circ}$ and $5^{\circ}$, respectively. Maximum protection of these movements and the use of correct systems for the problems that may occur due to the transition of mobileimmobile parts are important (16).

The characteristics of an ideal instrument are as follows: It should provide anatomic reduction and anatomic contour of the spine and indirect decompression with distraction and correction of the neural canal. It should have stability and rigidity, which will provide fusion biomechanically and will not require external support. Technically, the application should be easy, and it should be tissue-compatible and found easily (3).

Corrosion has started to become a major problem in spinal implants, and many issues have been experienced increasingly in recent years, including the wear (most common), corrosion, and fracture of fixation screws or longitudinal rods (21). Villarraga et al. (21) have observed wear after one year in all three different metal systems in their study.

The FE model (L4-L5) used in the study was successfully validated in all three main planes via a comparison of its results with those of various published in vitro studies. Specifically, the model was validated based on the fact that the kinematic data predicted by the FE model in all segments were within the standard deviation or close to the average of the results of in vitro studies.

In this study, an intact spine, a rigid system fixed with a rod, and modular plate systems were evaluated at Flex, Ext, LB, and AR. PRSS and NSS were compared in terms of ROM exercises. NSS has provided movement advantages in minimal Flex (0.6194-0.5756), Ext (0.1792-0.1551), and AR (0.3046-0.161) compared to PRSS, though PRSS has provided movement advantages in LB (0.0159-0.0056) compared to NSS. They did not show a statistically significant superiority over one another in terms of limitations on movement created during the ROM exercises (Table I).

Both systems have provided the rigidity needed in the region to which they were applied for fusion. PRSS has provided advantages of minimal intactness in minimal Flex (88.5-87.7), Ext (95.2-94.4), and AR (95.9-92.2) compared to NSS, and NSS has provided advantages of being minimally intact in LB (99.8-99.9) compared to PRSS (Table II). A significant superiority was not observed as a result of these values (Table II).

In light of all the results of the FE analysis, the PRSS and NSS systems applied do not have a significant superiority over one another. Equivalent results in the limitation of movement and the rigidity allow the use of these systems for short-segment posterior spinal instrumentation with the same indications. 
Spinal instrumentations are among the medical supplies that are most discussed and developed in the last century. Authors have always worked on something better. Except for a few notions that are now certain, many techniques and application materials are controversial. There are no adequate studies in the literature on the use of plates and rods in transpedicular screw spinal systems. Although plate systems developed in response to rigid rods, which are conventional methods, have started to be used widely today, long-term results are unknown. The most important short-term advantage of plate usage is the easy merging of the system with transpedicular screw heads in the perioperative period, reducing the operation time and the amount and time of anesthesia received. The first priority in this process is patient comfort and maintaining everyday life at a level close to normal. Other factors are the comfort of the surgeon, the amount of bleeding in the surgical areas, the time of the anesthesia, and the cost.

\section{CONCLUSION}

In light of the results of the FE analysis, the PRSS and NSS systems applied do not have significant superiority over one another. There are no adequate studies in the literature on the use of plates and rods in transpedicular screw spinal systems. It is believed that the most important short-term advantage of plate usage provides superiority over the rod system.

\section{- REFERENCES}

1. Albee FH: Transplantation of a portion of the tibia into the spine for Pott's disease. JAMA 57: 885-886, 1911

2. Boucher $\mathrm{HH}$ : A method of spinal fusion. J Bone Joint Surg (Br) 41: 248-259, 1959

3. Clohisy JC, Akbarnia BA, Bucholz RD, Burkus JK, Backer RJ: Neurologic recovery associated with anterior decompression of spine fractures at the thoracolumbar junction (T12-L1). Spine 17:325-330, 1992

4. Cloward RB: History of posterior lumbar interbody fusion. Springfields: Charles C Thomas 58, 1982

5. Cotrel $Y$, Dubousset J: A new technic for segmental spinal osteosynthesis using the posterior approach. Rev Chir Orthop Reparatrice Appar Mot 70(6): 489-494, 1984 (In French)

6. Edwards WC: The sacral fixation device: A new alternative for lumbosacral fixation. Paper read at the meeting of the North American Spine Society, Laguna Niguel, California, 1985:882
7. Foster MR: A functional classification of spinal instrumentation. Spine J 5(6): 682-694, 2005

8. Freeman BL: Scoliosis and Kyphosis. In: Canale ST (ed), Campbell's Operative Orthopaedics, Vol: 2, $10^{\text {th }}$ ed. Philadelphia: Mosby, 2003:1751-1837

9. Hadra BE: Wiring the spinous processes in Pott's disease. Trans American Orthopedic Associated 4: 206-210, 1891

10. Hibbs RA: An operation for progressive spinal deformities. New York Medical Journal 93: 1013-1016, 1911

11. Humphries AW, Hawk WA, Berndt AL: Anterior fusion of the lumbar spine using an internal fixation device. J Bone Joint Surg (Am) 41: 371, 1959

12. King D: Internal fixation for lumbosacral fusion. J Bone Joint Surg 30: 560-565, 1948

13. Krag $M H$, Beynnon $B D$, Pope $M H$ : An internal fixator for posterior application to short segments of the thoracic, lumbar or lumbosacral spine. Design and testing. Clin Orthop Relat Res 203:75 -98, 1986

14. Louis R: Fusion of the lumbar and sacral spine by internal fixation with screw plates. Clin Orthop Relat Res 203:18-33, 1986

15. Magerl F: External skeletal fixation of the lower thoracic and upper lumbar spine. Current Concepts of External Fixation of Fractures. Springer Berlin Heidelberg Verlag, 353-366, 1982

16. Ozar E, Senol O, Uysal L, Taskın M: Adjacent segment instability and degeneration after posterior lumbar stabilisation. Düşünen Adam 19(4): 194-203, 2006 (In Turkish)

17. Robinson RA, Smith GW: Anterolateral cervical disc removal and interbody fusion for cervical disc syndrome. Bull John Hopkıns Hosp 96: 223, 1955

18. Rogers WA: Treatment of fracture-dislocation of the cervical spine. J Bone Joint Surg 24: 245-258, 1942

19. Steffe AD, Biscup RS, Sitkowski DJ: Segmental spine plates with pedicle screw fixation. Clin Orthop Relat Res 203:45-53, 1986

20. Venable CS, Stuck WG: Electrolysis controlling factor in the use of metals in treating fractures. JAMA 3:349, 1939

21. Villarraga ML, Cripton PA, Teti SD, Steffey DL, Krisnamuthy $\mathrm{S}$, Albert T, Hilibrand A, Vaccaro A: Wear and corrosion in retrieved thoracolumbar posterior internal fixation. Spine (Phila Pa 1976) 31(21):2454-2462, 2006

22. Zielke K, Von Strempel AV: Posterior lateral distraction spondylodesis using the two fold sacral bar. Clin Orthop Relat Res 203:151-158, 1986 